\title{
PESQUISA, DESENVOLVIMENTO E INOVAÇÃO EM UM PROJETO DE INICIAÇÃO CIENTÍFICA E TECNOLÓGICA DE LONGO PRAZO: O CASO DO TOTAL SKY IMAGER
}

\author{
DOI: 10.37702/2175-957X.COBENGE.2021.3581
}

Camila Tiodista de Lima - camilatiodista@gmail.com

Instituto Federal de Educação Ciência e Tecnologia de Pernambuco

Rua Sebastião Cavalcante 386

55200-000 - Pesqueira - PE

Jonatas Santos de Souza - jonatassouza.dm@gmail.com

Instituto Federal de Educação Ciência e Tecnologia de Pernambuco

Rua Prof. José Carlos B. de Lima 77

55200-000 - Pesqueira - PE

Bruna Raquel Alves Silva - brunahouse9@gmail.com

Instituto Federal de Pernambuco

Rua Vereador Sebastião Castor Galindo 42

55260-000 - Alagoinha - PE

Ygo Neto Batista - ygo@pesqueira.ifpe.edu.br

IFPE Instituto Federal de Educação Ciência e Tecnologia

BR232 Km 214

53000-000 - Pesqueira - PE

Alexandre Manoel de Farias - alexandre.farias@pesqueira.ifpe.edu.br IFPE

Avenida Sul Governador Cid Sampaio 5095

51150-010 - Recife - PE

Resumo: Este trabalho apresenta o projeto do Total Sky Imager no IFPE Campus Pesqueira e seu impacto nos programas de iniciação científica e tecnológica do instituto. O Total Sky Imager (TSI) é um equipamento que captura imagens do céu e, a partir de um banco de conhecimentos e rede neural artificial, estima a irradiância solar a curto prazo. O projeto proposto foi iniciado em 2017 e está em andamento. Com potencial de gerar quarenta e oito planos de trabalho distintos, 0 
projeto envolve implementação em Android, Arduino, Matlab, entre outras ferramentas; de algoritmos de processamento de imagens, sistemas de controle, banco de dados, entre outros. Durante o período em que vem sendo desenvolvido, o projeto tem despertado aos alunos colaboradores o interesse na busca por soluções tecnológicas para os problemas do mundo moderno, solidificando conhecimentos adquiridos na sala de aula e agregando novos conhecimentos a sua formação acadêmica e profissional.

Palavras-chave: Total Sky Imager. PIBIC. projetos científicos. 


\section{PESQUISA, DESENVOLVIMENTO E INOVAÇÃO EM UM PROJETO DE INICIAÇÃO CIENTÍFICA E TECNOLÓGICA DE LONGO PRAZO: O CASO DO TOTAL SKY IMAGER}

\section{INTRODUÇÃO}

Iniciação Científica (IC) é a vinculação de alunos de graduação a projetos de pesquisa coordenados por professores experientes. A IC permite a integração dos estudantes com atividades e conteúdo não vistos no curso de graduação. Pode ser vista como "um excelente instrumento educativo que caminha entre a pesquisa e o ensino" (Bridi, 2004).

As instituições de ensino superior, seja por meio de bolsas próprias ou em parcerias com agências de fomento estaduais e federais, financiam alunos, bem como proporciona a estrutura para a realização dessas pesquisas. Em geral, o programa PIBIC (Programa de Institucional de Bolsas de Iniciação Científica) é a principal porta de entrada para os estudantes no universo da pesquisa. "Existe um consenso sobre os importantes objetivos alcançados com o PIBIC: o despertar e incentivo a vocação científica dos graduandos, além de favorecer a ampliação e a consolidação de núcleos de pesquisa nas IES." (Massi e Queiroz, 2010).

Outros meios de ingresso na IC é o PIBITI (Programa Institucional de Bolsas de Iniciação em Desenvolvimento Tecnológico e Inovação), BIA (Bolsa de Incentivo Acadêmico), PIBID (Programa Institucional de Bolsas de Iniciação à Docência), PET (Programa de Educação Tutorial), empresa júnior, etc. Esse vínculo de pesquisa também pode ser voluntário, a depender da disponibilidade e do interesse do aluno.

Ser um estudante pesquisador possibilita vantagens como introdução do aluno ao mundo da pesquisa, networking com professores e outros pesquisadores, além de ser uma opção de carreira profissional. É um excelente instrumento educativo na medida em que leva os alunos a lidarem com o processo de conhecer e não apenas com o produto desse processo. Os grupos de pesquisa normalmente vão muito além do conteúdo tratado em sala de aula, fator que agrega na formação e na construção de um currículo mais versátil. Na perspectiva do aluno que não tem a oportunidade de participar de atividades como o PIBIC, pode existir um déficit na compreensão do que é de fato e como funciona a pesquisa científica e, se esse estivesse incluso em atividades como essa, poderia descobrir novas habilidades, ter experiencia com esse tipo de atividade ou talvez descobrir na pesquisa uma opção de carreira.

Especificamente nos projetos desenvolvidos no IFPE Campus Pesqueira, em geral, estes são de curta duração, os quais normalmente tem duração de um ano e, ao final desse período, é apresentado um relatório com os principais resultados da pesquisa. No IFPE, geralmente, o professor precisa escrever projetos novos para participar dos editais de seleção. E nem sempre há a disponibilidade de tempo do docente visto as demais atividades acadêmicas, sobretudo de ensino, o que causa a interrupção das atividades de pesquisa científica.

O que será apresentado neste artigo é uma experiência do IFPE Campus Pesqueira em desenvolver um projeto de longo prazo, com capacidade para envolvimento de 48 estudantes do curso de Engenharia Elétrica em programas PIBIC, PIBIT ou BIA. O projeto 
em questão trata do desenvolvimento de um sistema de previsão de irradiância solar a curto prazo para aplicação em usinas solares.

A disponibilidade de energia solar está diretamente relacionada à cobertura de nuvens no céu. Em poucos segundos, é possível que a disponibilidade energética seja severamente alterada devido ao posicionamento das nuvens. Esta oscilação de disponibilidade tem como consequência redução da eficiência energética de usinas solar, inclusive da usina atualmente instalada no Campus Pesqueira. Existem equipamentos, denominados Total Sky Imager, capazes de elaborar previsões de curto prazo e, deste modo, melhorar a eficiência da usina.

O desenvolvimento de um Total Sky Imager de baixo custo no instituto, baseado em utilizar a câmera de um smartphone como sensor óptico e unidade de processamento, representa ganho de eficiência nas usinas do Campus e, com a difusão do conhecimento, em outras usinas no Brasil e no exterior.

\section{JUSTIFICATIVA}

Em abril de 2015, passou a funcionar no Campus Pesqueira uma usina geradora solar fotovoltaica. O campus possui uma área de $4.141 \mathrm{~m}^{2}$ para a montagem de sistemas de solo, com e sem rastreadores. Tais sistemas são utilizados na realização de práticas. Dispõe de 18 (dezoito) sistemas fotovoltaicos completos, que também são utilizados em pesquisas científicas na área de análise de defeitos, efeitos de condições ambientais, sujeiras e comissionamento. Recentemente o campus foi selecionado em concorrido edital pela Secretaria de Educação Profissional e Tecnológica (SETEC), Ministério da Educação (MEC), por meio do Programa para Desenvolvimento em Energias Renováveis e Eficiência Energética para a Rede Federal de Educação Profissional, Científica e Tecnológica (Programa EnergIF), para receber um Centro de Referência em Energia Solar Fotovoltaica (FV), destinado a contribuir para a capacitação de profissionais na área de Energia Solar Fotovoltaica.

Uma das linhas de atuação do Grupo de Pesquisa diz respeito ao desenvolvimento de protótipos de sistemas para aquisição, armazenamento e análise de parâmetros do ambiente (dados externos) ou fornecidos pelos módulos (dados internos) da usina solar fotovoltaica do Campus Pesqueira. Esses parâmetros são levantados a partir de medições realizadas por sensores e transdutores, são armazenados, tratados e analisados. Estudamos e relacionamos a influência da variação de parâmetros (internos e externos aos módulos) no desempenho dos módulos solares da usina do Campus Pesqueira e sugerimos alternativas para minimizar os efeitos negativos causados pela influência desses parâmetros.

Visto a infraestrutura disponível no IFPE e as linhas de pesquisa desenvolvidas, foi elaborado um projeto de pesquisa para desenvolvimento de um Total Sky Imager com perspectiva de execução de longo prazo, estimado inicialmente em oito anos de execução, e capacidade de fornecer planos de trabalho para 48 estudantes do curso de Engenharia Elétrica do Campus Pesqueira.

\section{FUNDAMENTAÇÃO TEÓRICA}

Um dos equipamentos mais relevantes em desenvolvimento pelo grupo é o Total Sky Imager (TSI). A disponibilidade de energia solar está diretamente relacionada à cobertura de nuvens no céu. Em poucos segundos, é possível que a disponibilidade energética seja severamente alterada devido ao posicionamento das nuvens. Esta oscilação de disponibilidade tem como consequência redução da eficiência energética de usinas solar, 
inclusive da usina atualmente instalada no IFPE Campus Pesqueira. Existem equipamentos, denominados Total Sky Imager, capazes de elaborar previsões de curto prazo e, deste modo, melhorar a eficiência da usina (Nouri, 2019; Valentín, 2019; Tang, 2019; Ryu, 2019). Contudo, tais equipamentos tem custo elevado e não há evidências que existem muitas unidades no Brasil.

O desenvolvimento de um Total Sky Imager de baixo custo no IFPE, baseado em utilizar a câmera de um smartphone como sensor óptico, com lentes e filtros externos e utilizando o sistema operacional Android, representará ganho de eficiência na usina do IFPE e, com a difusão do conhecimento, em outras usinas no Brasil e no exterior. Existem outras pesquisas no mundo em relação ao desenvolvimento de TSI com baixo custo, como o descrito por Valentín (2019) ou o de Nouri (2019), porém com configurações diferentes da proposta neste projeto.

Contudo, o caminho técnico a ser percorrido é longo. O desenvolvimento do Total Sky Imager no IFPE foi iniciado em 2017 através de 03 (três) planos de trabalhos apresentados e aprovados no edital interno PIBIC. Em 2018 tivemos 01 (um) estudante BIA FACEPE e mais 03 (três) PIBIC IFPE. Em 2019, tivemos mais 03 (três) PIBIC internos. Em 2020, foram 03 (três) PIBIC, sendo um deles financiado pela FACEPE. E, neste ano, pleiteamos mais 3 bolsas, sendo 02 à FACEPE, e 1 para o IFPE, visto a redução drástica da disponibilidade financeira do IFPE para bolsas PIBIC.

\section{METODOLOGIA}

É possível observar na Figura 1 um diagrama que detalha a execução do projeto. Na etapa de levantamento de requisitos (1.1), levantamos os requisitos mínimos para o desenvolvimento do protótipo. A partir dos requisitos, iniciamos o desenvolvimento de cada atividade de acordo com o diagrama apresentado, sempre com planos de trabalho independentes para cada atividade. Foram definidas as interfaces de modo que os planos de trabalho podem ser executados em paralelo em sua maioria, pelos estudantes trabalhando remotamente na maioria dos casos, o que é adequado para um cenário pandêmico.

Cada plano de trabalho é executado de acordo com o modelo espiral simples. $\mathrm{O}$ modelo espiral descreve um fluxo de atividades cíclico e evolutivo, em quatro estágios: 1. Determinação dos objetivos, alternativas e restrições; 2. Avaliação das alternativas, identificação e resolução de riscos; 3. Implementação do sistema; 4. Avaliação dos resultados e planejamento do próximo ciclo.

A integração entre os planos de trabalho em andamento é realizada através de reuniões semanais com toda a equipe. Todos os estudantes acompanham a execução de todos os planos de trabalho. Além de melhorar a fluidez da integração entre planos, que já tinha interfaces clara entre eles, também multiplica o repertório de ferramentas e algoritmos adquiridos pelos estudantes. Até o presente momento, cada estudante envolvido executou, em média, dois planos de trabalho. No início de cada ciclo, principalmente quando há novos estudantes, realizamos uma revisão de tudo o que já foi desenvolvido e um treinamento nas principais ferramentas que serão utilizadas. Este treinamento é propiciado pelos estudantes veteranos, para os estudantes novatos. Durante a execução do projeto, as dificuldades enfrentadas pelos estudantes são esclarecidas durante as reuniões semanais. Caso seja uma dificuldade que demande mais tempo para esclarecimento, é marcada uma reunião com o professor orientador em separado e, após a resolução do problema, um resumo é apresentado para os demais, de modo que todos sempre estão cientes dos algoritmos em desenvolvimento. 
A respeito da implementação do sistema, está sendo utilizado a IDE Android Studio, que é a interface mais utilizada para desenvolver aplicativos Android. A linguagem de programação é o Java, visto que esta linguagem é estudada no 4 o período do curso de Engenharia Elétrica, e os estudantes candidatos a bolsa PIBIC já cursaram tal disciplina.

Figura 1 - Diagrama do Projeto.

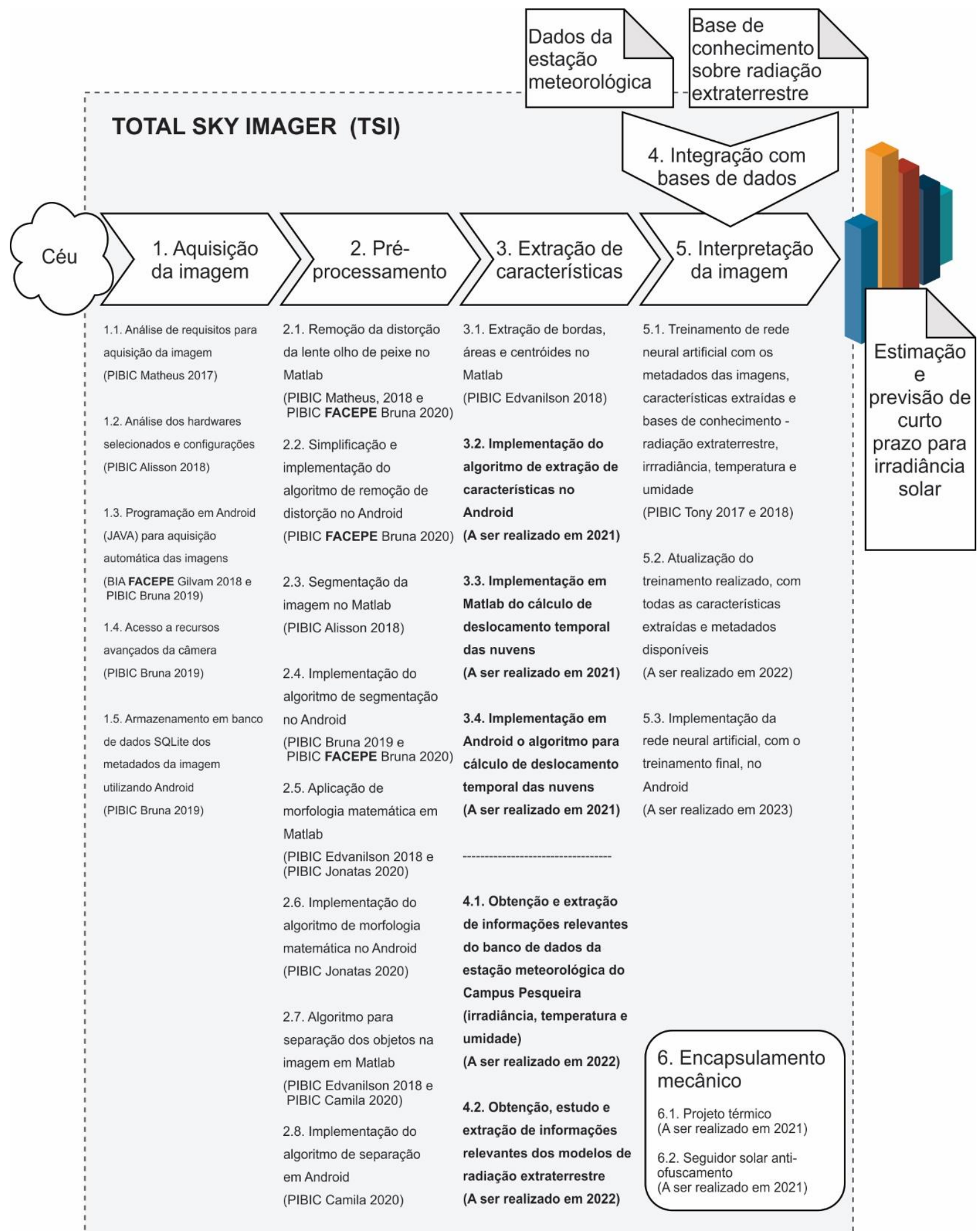

Fonte: autores. 
A automação do sistema anti-ofuscamento e de controle de temperatura é feito utilizando o Arduino. Quanto a infraestrutura, é necessário apenas o uso de computadores para os Objetivos de 1 a 5. O IFPE Campus Pesqueira possui 3 (três) laboratórios de informática e 1 (um) laboratório de eletrônica com computadores, possuindo ampla disponibilidade de uso para execução das atividades relacionadas a este projeto. Relacionado ao objetivo 6, está disponível também a oficina de mecânica e manutenção do Campus Pesqueira. O laboratório de eletrônica possui todos os equipamentos e ferramentas necessárias para o desenvolvimento das atividades planejadas para esse objetivo 6. A partir do próximo ano estará disponível também um laboratório de novas tecnologias / indústria 4.0, o qual dispõe de kits de microcontroladores, realidade aumentada, impressão 3D, CNC, entre outros.

A execução de cada atividade é precedida de um estudo acadêmico relacionado aos algoritmos já implementados e testados em outros grupos de pesquisa e um estudo técnico relacionado ao uso das ferramentas computacionais necessárias (linguagens de programação e ambientes de desenvolvimento). Adicionalmente, os estudantes precisam estudar a linguagem de programação MatLab, bem como as ferramentas da interface integrada de desenvolvimento.

Relativo aos planos de trabalho já executados, tivemos bons resultados técnicos. O Objetivo 1, aquisição de imagens, foi completamente concluído através de 03 (três) trabalhos PIBIC e 01 (um) BIA FACEPE. Apesar que o software pode ser melhorado ainda, abrindo possibilidade para novos planos de trabalho. Os algoritmos referentes ao Objetivo 2 foram completamente desenvolvidos e implementados através de 06 (seis) trabalhos PIBIC, sendo um financiado pela FACEPE.

Quanto às implementações para alcançar os objetivos 3 e 4, apenas a extração de bordas, áreas, centróides foi realizada no Matlab através de 01 (um) trabalho PIBIC. Por fim, relativo ao objetivo 5, 01 (um) trabalho PIBIC foi o estudo de redes neurais artificiais e implementação de um modelo, porém, como não havia uma base de dados consistente na época, não foi possível obter os resultados esperados. O objetivo 5 é o que mais depende dos resultados anteriores. O projeto mecânico já foi idealizado, referente ao objetivo 6 , porém ainda não implementado uma vez que consideramos mais adequada a execução das atividades 6.1 e 6.2 após a implementação dos Objetivos 1 e 2, ou seja, a partir deste ano.

\section{RESULTADOS E DISCUSSÃO}

O Total Sky Imager, em seu quinto ano de desenvolvimento, tem rendido muitos frutos. Relativo às publicações, trabalhos foram divulgados nos CONICs 2018, 2019 e 2020 e em 03 (três) trabalhos foram apresentados no Congresso Norte Nordeste de Pesquisa e Inovação (CONNEPI 2019). Porém, o maior potencial de publicação é quando o sistema estiver completamente pronto. Este desenvolvimento abre novas possibilidades para publicações científicas, registro de propriedade intelectual através de patente e incubação do projeto.

Durante esse período de mutualidade entre pesquisador e o objeto de pesquisa, treze bolsas PIBIC foram implementadas para o seu desenvolvimento. Ao desenvolver o projeto, o bolsista conta com a oportunidade de conhecer e aprofundar seus conhecimentos em temas como: processamento de imagens, programação em diferentes linguagens, desenvolvimento de aplicações para Android, Arduino, GitHub, entre outros. Tais temas proporcionam melhor desenvolvimento acadêmico, além de acrescentar um diferencial ao currículo profissional do estudante. 
Para o bolsista PIBIC, desenvolver pesquisa no âmbito da Iniciação Científica faz o estudante se familiarizar com grupos de pesquisas no IFPE e externos, tal como o Grupo de Elétrica de Potência e Acionamentos Elétricos (GEPAE), da UFPE. O projeto tem proporcionado aos estudantes a aprendizagem de técnicas e métodos científicos, bem como estimula o desenvolvimento do pensar cientificamente e da criatividade, decorrentes das condições criadas pelo confronto direto com os problemas de pesquisa. Atualmente o projeto conta com a colaboração de três bolsistas PIBIC, trabalhando na finalização das etapas de implementação em Android. Para o próximo ano já é levantado a possibilidade da implementação de mais quatro bolsas PIBIC para finalização de mais duas etapas.

A seguir, relatos de alguns estudantes que participaram ou participam deste projeto a fim de possibilitar ao interlocutor um melhor entendimento sobre os benefícios que a iniciação científica proporciona no campo acadêmico, além de esclarecer em que aspectos o projeto influenciou suas maneiras de pensar o curso e estabelecer objetivos para o futuro profissional.

Segundo o estudante Jônatas Santos, "Ter essa experiencia com a pesquisa cientifica está sendo muito importante, visto que me trouxe a oportunidade de colocar em prática conteúdos estudados em disciplinas regulares da graduação e incentiva explorar outras áreas do conhecimento, que amplia a visão do mundo além da que a formação acadêmica proporciona. No ponto de vista pessoal nada melhor do que ter a oportunidade de conhecer e interagir com outros pesquisadores, sejam eles discentes ou docentes, que compartilham os mesmos interesses de pesquisa que você."

Camila Tiodista afirma que "A pesquisa além de trazer conhecimento técnico me ensinou a forma de aprender autônoma, com o professor apenas direcionando e tirando dúvidas. A oportunidade de colocar em prática conhecimentos adquiridos na disciplina Programação Orientada a Objeto atrelado a prática em si, com teorias que vão além da programação, como a interpretação das imagens."

Segundo Bruna Raquel, "Minha participação no projeto acrescentou positivamente na minha experiência acadêmica e também pessoal, pois foi uma grande honra estar inserida numa pesquisa científica que reveste os caminhos futuros e se propõe a demonstrar como o nosso mundo pode vir a ser e funcionar."

Como visto, um projeto como o TSI acrescenta na educação dos estudantes de uma forma singular, permitindo o sentimento de inclusão, além de auxiliar a traçar expectativas para o futuro, entretanto os benefícios não se estendem tão somente aos estudantes.

Do ponto de vista social, são gerados conhecimentos científicos e recursos tecnológicos no campo do monitoramento e controle da geração de energia solar capazes de contribuir para o desenvolvimento sustentável local e regional, integrando ensino, pesquisa e extensão, em perfeita sintonia com a missão institucional do IFPE.

Do ponto de vista do instituto, considerando a importância de estudos e pesquisas com o objetivo de viabilizar cada vez mais a inserção de fontes de energia alternativas e renováveis, e observando o grande potencial para pesquisa e investigação científica latente no corpo docente e discente da instituição, o projeto beneficia o processo de consolidação do Grupo de Pesquisa Interdisciplinar em Energias Renováveis e Sistemas Eletroeletrônicos do Campus Pesqueira. 
O Total Sky Imager inegavelmente tem sido relevante na formação acadêmica e profissional dos alunos do IFPE Campus Pesqueira. Seu desenvolvimento tem guiado cada vez mais o aluno na descoberta de novos horizontes tecnológicos e científicos, com intuito de solucionar problemas propostos pelo mundo moderno. Na troca mútua de saberes entre pesquisador e objeto de pesquisa, o desenvolvimento do TSI tem agregado ao bolsista novos conhecimentos, como também proporcionado o aprofundamento em temas vistos em sala de aula, desta forma o aluno presencia no ato de pesquisar a utilidade prática de tais, no meio científico e tecnológico.

O projeto segue em fase de desenvolvimento, e a cada ano tem inserido novos alunos ao contexto da iniciação cientifica, com o potencial de envolvimento de 48 (quarenta e oito) planos de trabalho distintos. A partir do momento que o projeto estiver com todas suas etapas concluídas, o TSI será uma ferramenta econômica e eficaz de grande importância para usina solar do IFPE. Após o projeto concluído, será demandado apoio do NIT (Núcleo de Inovação Tecnológica) do IFPE para estudar as possibilidades relacionadas a proteção intelectual e industrial do sistema desenvolvido.

\section{REFERÊNCIAS}

BRIDI, J. C. A. A Iniciação científica na formação do universitário. 2004. Dissertação (Mestrado em Educação) - Faculdade de Educação, Universidade Estadual de Campinas, Campinas.

MASSI, L.; QUEIROS L. S. Estudos sobre iniciação científica no Brasil: uma revisão. Translated by Robert Dinham. Cad. Pesqui. [online]. 2010, vol.40, n.139, pp.173-197.

NOURI, Bijan et al. Determination of cloud transmittance for all sky imager based solar nowcasting. Solar Energy, v. 181, p. 251-263, 2019.

RYU, Anto et al. Preliminary Analysis of Short-term Solar Irradiance Forecasting by using Total-sky Imager and Convolutional Neural Network. In: 2019 IEEE PES GTD Grand International Conference and Exposition Asia (GTD Asia). IEEE, 2019. p. 627-631.

TANG, Jiacheng et al. An improved cloud recognition and classification method for photovoltaic power prediction based on total-sky-images. The Journal of Engineering, $v$. 2019, n. 18, p. 4922-4926, 2019.

VALENTÍN, Luis et al. Towards the Development of a Low-Cost Irradiance Nowcasting Sky Imager. Applied Sciences, v. 9, n. 6, p. 1131, 2019. 


\title{
RESEARCH, DEVELOPMENT AND INNOVATION IN A LONG TERM SCIENTIFIC AND TECHNOLOGICAL INITIATION PROJECT: THE CASE OF TOTAL SKY IMAGER
}

\begin{abstract}
This work presents the Total Sky Imager project at IFPE Campus Pesqueira and its impact on the institute's scientific and technological initiation programs. Total Sky Imager (TSI) is a device that captures images from the sky and, based on a knowledge base and artificial neural network, estimates the solar irradiance in the short term. The proposed project started in 2017 and is ongoing. With the potential to generate forty-eight distinct work plans, the project involves implementation on Android, Arduino, Matlab, among other tools; image processing algorithms, control systems, database, among others. During the period in which it has been developed, the project has aroused the interest of collaborating students in the search for technological solutions to the problems of the modern world, strengthening the knowledge acquired in the classroom and adding new skills to their academic and professional training.
\end{abstract}

Keywords: Total Sky Imager, PIBIC, Scientific projects. 